\title{
Fístula Carótido-Cavernosa con resolución quirúrgica; Un reporte de caso y revisión de literatura.
}

\section{Carotid cavernous fistula with surgical resolution; a case report and literature review.}

Pág. 12,17

Recibido: 07-05-2020

Aceptado: 08-06-2020

Dr. Mora Navarro Daniel ${ }^{1}$

Dr. Biehl Saborio Franz ${ }^{2}$

1. Médico General,Trabajador independiente, Costa Rica.

2. Médico General,Trabajador independiente, Costa Rica.

\section{RESUMEN}

Las fístulas carótido cavernosas son comunicaciones anormales entre el sistema arterial carotideo y el seno venoso cavernoso. Estas son adquiridas y de inicio tanto espontaneo como crónico. Divididas anatómicamente en directas donde su etiología principal es traumática, como dúrales donde la etiología es multifactorial. Se presenta el caso de un paciente portador de una fístula carótido-cavernosa tipo C. Un hombre de 59 años conocido sano inicia con sintomatología 7 meses después de un trauma penetrante a cuello anterior. Inyección conjuntival, dolor en hemicara, diplopía y tinnitus pulsátil. Se realizan múltiples tomografías computarizadas y una resonancia, las cuales orientan a un diagnóstico vascular, el cual es corroborado con una angiografía, la cual logra diagnosticar una fístula carótido cavernosa tipo C. Esta fue tratada mediante embolización endovascular en dos ocasiones con disminución de la sintomatología y normalización de la presión intraocular.

\section{PALABRAS CLAVE}

Fístula carótido cavernoso, inyección conjuntival, trauma, cefalea pulsátil, seno cavernoso, arteria carótida.

\section{ABSTRACT}

Carotid cavernous fistulas are abnormal communications between the carotid arterial system and the venous cavernous sinus. These can be divided anatomically into direct, where the main etiology is traumatic, and dural where the etiology is multifactorial. Presented here is a case of a patient with a type $C$ carotid cavernous fistula. The case of a 59-year-old male previously healthy presents conjunctival 
injection, hemifacial pain, diplopia, and pulsatile tinnitus 7 months after penetrating anterior neck trauma. Several CT scans and MRI are performed which guide the diagnosis towards a vascular origin. An angiography finally establishes the diagnosis of a type $\mathrm{C}$ carotid cavernous fistula. An embolization is performed in two occasions which normalizes intraocular pressure and reduces symptomatology.

\section{KEYWORDS}

Carotid cavernous fistula, conjunctival injection, trauma, throbbing headache, cavernous sinus, carotid artery.

\section{REPORTE DE CASO}

Se presenta el caso de un paciente masculino de 59 años conocido sano con antecedente de trauma penetrante de cuello anterior, 7 meses antes del inicio de síntomas. Trauma ocurrido con un fragmento de alambre de púa el cual viaja como proyectil al ser impactado con una moto guadaña.

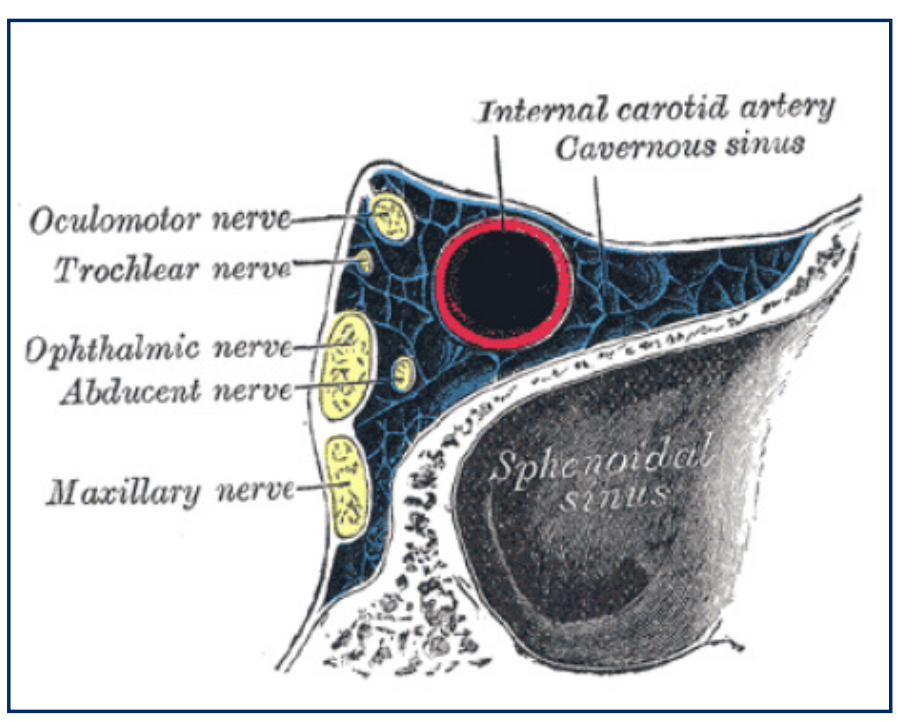

Figura 1. Anatomía del seno cavernoso (Wikimedia commons, Grays anatomy)

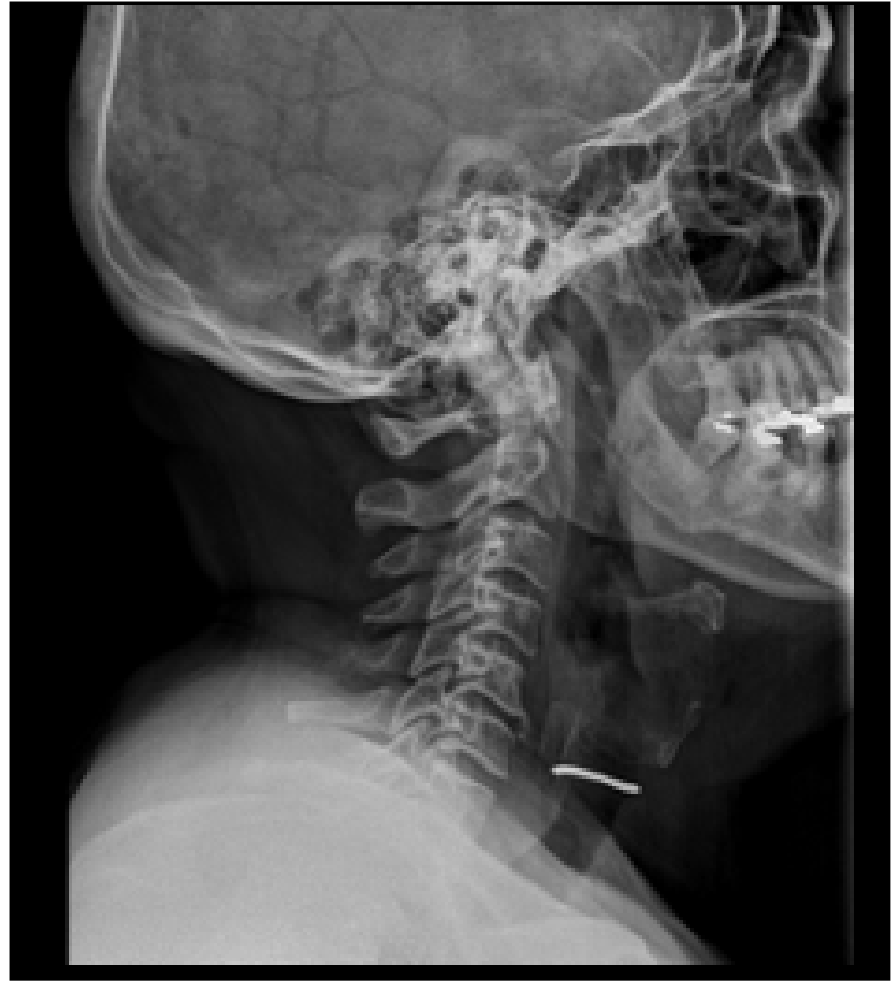

Figura 2. Radiografía de cuello lateral. Se observa cuerpo extraño anterior a vía aérea.

(Base de datos Radiologia Hospital Mexico., 2019)

Intraoperatoriamente durante la laringoscopía con traqueostomía se realiza hallazgo de un cuerpo extraño metálico posterior a istmo tiroideo hasta región subglótica a nivel submucoso. Se realiza cierre de traqueostomía y el paciente se egresa sin complicaciones y, continúa en control por la consulta externa. Durante los controles, el paciente no aqueja ninguna sintomatología.

El paciente inicia con sintomatología 5 meses después. Aqueja dolor en hemicara derecha con paresias e inyección conjuntival bilateral con diplopía y dolor al realizar movimientos oculares como síntomas principales, dada la persistencia de su sintomatología a pesar de tratamiento sintomático se realiza estudios por privado. Se documenta por medio de una 
tomografía contrastada un quiste aracnoideo vs mega cisterna en fosa posterior como hallazgo incidental. Pero sin datos de malformación arteriovenosa ni dilataciones aneurismáticas. Se realiza una resonancia magnética encontrándose un "Aumento del volumen de los tejidos blandos del seno cavernoso izquierdo, principalmente (siendo el de mayor engrosamiento el seno izquierdo, mostrando realce homogéneo que se proyecta a la fisura orbitaria superior."

La sintomatología avanza hasta presentar quemosis con leve edema periorbitario con midriasis y exoftalmos bilateral no cuantificado. Asimismo, inicia con acúfenos pulsátiles que fluctúa entre oídos y dolor submaxilar izquierdo.

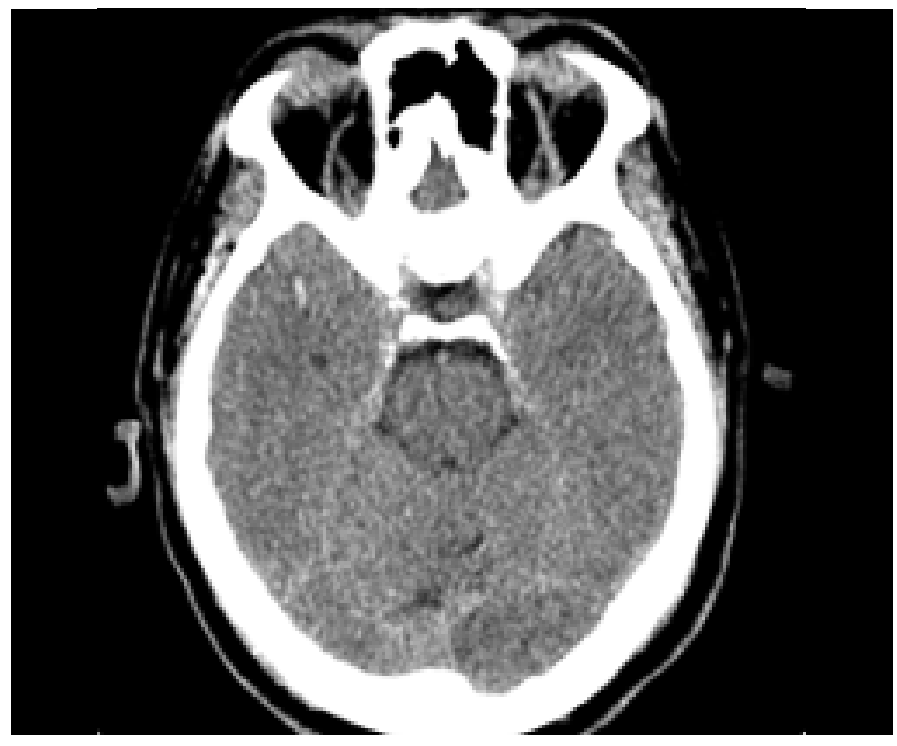

Figura 3. T.A.C. Se observa aumento de diámetro de vena oftálmica superior izquierda. Realce de senos cavernosos. (Base de datos Radiologia Hospital Mexico., 2019)

Se realiza una tonometría por aplanación con una PIO (presión intraocular) de $22 \mathrm{~mm} \mathrm{Hg}$ OD y $24 \mathrm{~mm} \mathrm{Hg}$ OS con agudeza visual simétrica 20/25 con pinhole. Fondo de ojo: Nervio óptico 0.5 Excavación íntegra, Macula sin lesiones, Retina aplicada en 4 cuadrantes.
Se realiza agio-T.A.C de cerebro el cual se reporta como:

"Sin franca evidencia de lesiones aneurismáticas ni fístulas. Asocia reforzamiento de medio de contraste a nivel de seno cavernoso."

Neurocirugía realiza intervención quirúrgica con angiografía y embolización de pedículos de arteria meníngea media y maxilar izquierda y faríngea ascendente derecho, con lo cual se diagnostica una fístula carótida cavernosa tipo C.

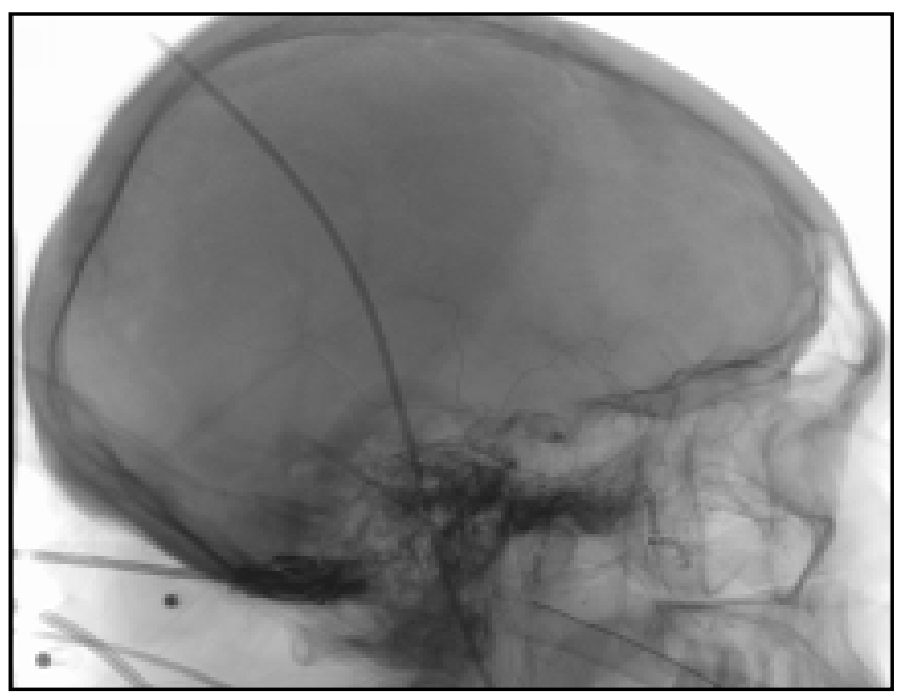

Figura 4. Angiografía cerebral. Se observa Ilenado de medio de contraste por la ACE derecha con difuminación del medio de contraste en el sitio de la fístula. Lo cual define la fístula como de bajo flujo.

Una segunda angiografía confirmatoria observa persistencia de la fístula y se embolizan pedículos de maxilar interna y meníngea media derecha. Persisten pedículos de ramas de arterias carótidas internas. Estos no se embolizan.

Se confirma una PIO postoperatoria OD $20 \mathrm{~mm} \mathrm{Hg}$, OS $22 \mathrm{~mm} \mathrm{Hg}$. Ambos ojos con persistencia de vasos congestivos y ausencia de sintomatología reportada por el paciente. 


\section{DISCUSIÓN}

Las fístulas carótido cavernosas son malformaciones arteriovenosas adquiridas, en vez de congénitas. Estas se pueden dividir en malformaciones de alto flujo y malformaciones de bajo flujo. Las lesiones de alto flujo se presentan cuando existe una comunicación directa entre la arteria carótida y los senos venosos durales. Las lesiones de bajo flujo se presentan como una fístula dural que permite comunicación indirecta entre el seno cavernoso con ramas de la carótida interna o carótida externa.

La clasificación de Barrow es la utilizada actualmente para subclasificar las fístulas carótido-cavernosas. Esta clasifica las malformaciones de alto flujo como $A$. Las fístulas carótido-cavernosas tipo A se asocian con una única laceración endotelial de la carótida, que permite flujo directamente hacia el seno cavernoso. Las categorías B-D son de bajo flujo. En la categoría $B$, el flujo arterial proviene de ramas pequeñas de la porción cavernosa de la arteria carótida. En la categoría C, la presente en el paciente de este caso, el flujo arterial proviene de ramas de la carótida externa. Esto se comprueba en la angiografía con embolización de ramas de la arteria meníngea media y maxilar izquierda. Por último, las fístulas clasificadas como tipo $D$, tienen un componente mixto entre aferencias provenientes de la arteria carótida interna y carótida externa.

La incidencia de esta patología no se encuentra documentada con exactitud en la literatura al momentodela revisión deestecaso. Enunestudio alemán de 1994, acerca de la fisiopatología de las fístulas carótido-cavernosas llevada a cargo por Helmke $\mathrm{K}$, se evidenció que la patología es igualmente prevalente en mujeres que en hombres y, que se puede presentar en cualquier grupo etario. En una investigación realizada el 2007, por Liang W, para la universidad de Zhejiang en China, se concluyó que las fístulas carótido-cavernosas pueden estar presentes en un $4 \%$ de los pacientes con fractura basilar del cráneo. En el contexto traumático, las fístulas carótido-cavernosas son más prevalentes en el sexo masculino. La hipótesis actual sugiere que el aumento de la presión arterial intraluminal causa ruptura espontánea, con la subsecuente creación de una fístula.

La etiología de las fístulas carótido-cavernosas no traumáticas es en su mayoría idiopática. Hay factores precipitantes comoloson colagenopatías como Ehlers-Danlos, aneurismas carotídeos previamente existentes y por comunicaciones arterio-durales microscópicas previamente existentes.

La clínica presente en paciente con fístulas carótido-cavernosas es muy variada. A continuación, se detalla las manifestaciones clínicas más comunes en fístulas tipo $A$ :

- $80 \%$ asocia sonidos de "flujo de agua"

- $29-50 \%$ asocia visión borrosa

- $53-75 \%$ asocia cefalea

- $50-85 \%$ asocia diplopía

- $35 \%$ asocia dolor orbicular

- $72-87 \%$ asocia proptosis

- 55-89\% asocia quemosis e inyección conjuntival

- Oftalmoplejía hasta en $85 \%$ de los casos

En las fístulas de bajo flujo o clasificaciones B-D, normalmente presentan menos sintomatología que las de alto flujo. En estos casos, los síntomas más comunes son molestias en el área orbitaria, dolor ocular, pérdida de visión. 
La pérdida de agudeza visual, generalmente se asocia a malformaciones anteriores con ramas de la arteria carótida interna. Esto se debe a una o varias de las siguientes causas:

- Aumento de la presión intraocular secundario a congestión orbitaria

- Retinopatía secundaria a estasis venosa

- Hemorragia vítrea

- Retinopatía proliferativa

- Neuropatía óptica isquémica

- Desprendimiento exudativo de retina

La reconstrucción angiográfica es el "gold standard" para el diagnóstico de fístula carótidocavernosa. No obstante, el TAC, la resonancia magnética y la angiografía convencional también son útiles para establecer el diagnóstico. En el TAC se busca expansión del seno cavernoso y aumento del volumen de la vena oftálmica superior. La resonancia magnética es más sensible para buscar datos de proptosis y alteraciones en el drenaje del seno cavernoso.

El tratamiento depende de varios factores. En fístulas de alto flujo, la probabilidad de resolución espontánea es muy baja y los pacientes que las presentan se encuentran generalmente sintomáticos. Por estas razones, se prefiere dar un manejo intervencionista. Las fístulas que presentan menos sintomatología pueden ser observadas de manera expectante. La sintomatología del paciente será lo que ultimadamente decidirá el curso del tratamiento. La obliteración endovascular es la técnica de elección para llevar a cabo este procedimiento. Esto se puede lograr mediante embolización transarterial 0 transvenosa. Varios estudios sugieren que se logra una reparación en hasta un $99 \%$ de los casos. El estudio realizado por Wang, W para la Universidad Shanghái Jiao Tong reporta que hasta un $84 \%$ de los pacientes tuvo una reparación completa con solo una técnica quirúrgica, ya sea con colocación de balones o con bobinas.

En paciente con fístulas de bajo flujo, la embolización transarterial se vuelve más difícil, por lo que se opta por embolización transvenosa. El manejo quirúrgico es reservado para pacientes donde no se logre un abordaje endovascular o este sea inútil.

\section{BIBLIOGRAFÍA}

1. Ellis JA, Goldstein BS, Connoly ES, Meyers PM (2012) Carotid-cavernous fístulas. Neurosurg Focus 32(5): E9

2. Liang W, Xiaofeng Y, Weiguo L, Wusi Q, Gang S, Xuesheng Z (2007) Traumatic carotid cavernous fístula accompanying basi-lar skull fracture: a study on the incidence of traumatic carotid cavernous fistula in the patients with basilar skull fracture and the prognostic analysis about traumatic carotid cavernous fís-tula. J Trauma 63:1014-1020.

3. De Keizer R (2003) Carotid cavernous and orbital arteriovenous fistulas: ocular features, diagnostics, and hemodynamic considerations in relation to visual impairment and morbidity. Orbit 22:121-142.

\section{(@)}


4. Kirsch M, Henkes H, Liebig T, Weber W, Esser J, Golik S, Kuhne D (2006) Endovascular management of dural carotid- cavernous sinus fistulas in 141 patients. Neuroradiology 48:486490.

5. Gupta AK, Purkayastha S, Krishnamoorthy T, Bodheey NK, Kapilamoorthy TR, Kesavadas C et al (2006) Endovascular treatment of direct carotid cavernous fistulae: a pictorial review. Neuroradiology 48:831-839.

6. Al-Mufti F, Amuluru K, El-Ghanem M et al (2017) Spontaneous bilateral carotid-cavernous fistulas secondary to cavernous sinus thrombosis. Neurosurgery 80:646-654.

7. Mason TH, Swain GM, Osherof HR (1954) Bilateral carotid-cavernous fistula. J Neurosurg 11:323-326

8. Jamieson KG (1964) Bilateral carotico-cavernous fistulae. Hypo-pituitarism from bilateral carotid ligation for surgical cure. Aust NZ J Surg 34:1-10.

9. Barrow DL, Spector RH, Braun IF, et al. Classification and treatment of spontaneous carotidcavernous sinus fistulas. J Neurosurg 1985; 62:248.

Helmke K, Krüger O, Laas R. The direct carotid cavernous fistula: a clinical, pathoanatomical, 10. and physical study. Acta Neurochir (Wien) 1994; 127:1.

11. Wang W, Li YD, Li MH, et al. Endovascular treatment of post-traumatic direct carotid-cavernous fistulas: A single-center experience. J Clin Neurosci 2011; 18:24.

12. Meyers PM, Halbach VV, Dowd CF, et al. Dural carotid cavernous fistula: definitive endovascular management and long-term follow-up. Am J Ophthalmol 2002; 134:85.

13. Base de datos Radiología Hospital México. (2019). la uruca, San Jose, Costa Rica.

14. Wikimedia commons. (Gray's anatomy). From Wikimedia commons: https://upload.wikimedia. org/wikipedia/commons/d/d9/Gray786.png 